\title{
Revisão da literatura: Acompanhamento farmacoterapêutico prestado aos pacientes diabéticos do tipo 2 atendidos em farmácias comunitárias
}

\author{
Literature review: Pharmacotherapeutic follow-up provided to type 2 diabetic patients seen at \\ community pharmacies
}

Revisión de la literatura: Seguimiento farmacoterapéutico proporcionado a pacientes diabéticos tipo 2 atendidos en farmacias comunitárias

Juan Gonzalo Bardález Rivera ORCID: https://orcid.org/0000-0003-1737-6947 Escola Superior da Amazônia, Brasil E-mail: jgrivera@bol.com.br

Gleicy Kelly China Quemel ORCID: https://orcid.org/0000-0003-1280-560X Escola Superior da Amazônia, Brasil E-mail: gkcquemel@gmail.com

Viviane Monteiro da Silva ORCID: https://orcid.org/0000-0002-5136-2402 Instituto Evandro Chagas, Brasil

E-mail: vivianemonteiro95@outlook.com Jéssica Giselli da Costa ORCID: https://orcid.org/0000-0001-7126-2241 Escola Superior da Amazônia, Brasil E-mail: hendmoda90@gmail.com

Kezia Rayane Paulino da Silva ORCID: https://orcid.org/0000-0001-9420-3375 Escola Superior da Amazônia, Brasil E-mail: kezia.rayane13@gmail.com

Jonatas Borges Costa

ORCID: https://orcid.org/0000-0003-3780-9439 Escola Superior da Amazônia, Brasil E-mail: jonatasborgescosta9@gmail.com

\begin{abstract}
Resumo
Esta revisão tem como objetivo apresentar evidências, com base na literatura, quanto à relevância do acompanhamento farmacoterapêutico prestado aos pacientes com diabetes mellitus tipo 2 atendidos em farmácias comunitárias, além de conhecer o papel do farmacêutico comunitário no controle do diabetes tipo 2 e investigar os pontos positivos do acompanhamento farmacoterapêutico destinados a pacientes diabéticos. Trata-se de uma revisão de literatura realizada por meio de busca de artigos originais em bases de dados como Scielo, PubMed e Lilacs. Os critérios de inclusão foram: publicações que atenderam a proposta da temática, artigos completos, disponíveis em língua portuguesa e publicados no período de 2010 a 2019. Os critérios de exclusão foram: artigos pagos e duplicados, assim como estudos incompletos, que não atenderam ao objetivo e temática da pesquisa, além de livros, dissertações e teses. Foram selecionados três artigos que compuseram a revisão e evidenciaram que as principais dúvidas dos pacientes portadores de DM estavam relacionadas aos horários de administração dos medicamentos. Outros achados mostraram que houve boa aceitabilidade da proposta de seguimento farmacoterapêutico e que sua aplicabilidade em farmácias comunitárias privadas traz diversos benefícios como maior adesão ao tratamento, ocorrendo de forma racional com melhoria dos resultados clínicos e estabelecimento de vínculo entre farmacêutico e usuário, reafirmando a capacidade do farmacêutico para a realização das atividades clínicas no setor privado. Este estudo tornou evidente que a prática da assistência farmacêutica e das boas práticas de dispensação direcionadas aos pacientes atendidos em drogarias pode ser alcançada sem a necessidade de grandes alterações estruturais no estabelecimento e sem a contratação adicional de funcionários.
\end{abstract}

Palavras-chave: Diabetes mellitus tipo 2; Acompanhamento farmacoterapêutico; Farmacêutico comunitário.

\section{Abstract}

This review aims to present evidence as to the relevance of pharmacotherapeutic monitoring provided to patients with type 2 diabetes mellitus treated in community pharmacies, in addition to knowing the role of community pharmacists 
in the control of type 2 diabetes and investigating the positive aspects of pharmacotherapeutic monitoring aimed at diabetic patients. This is a literature review carried out by searching for original articles in databases such as Scielo, PubMed and Lilacs. The inclusion criteria were: publications that met the proposal of the theme, complete articles, in portuguese and published from 2010 to 2019. The exclusion criteria were: incomplete studies, which did not meet the objective and theme of the research, in addition to books, dissertations and theses. The articles that made up the review showed that the main doubts of patients with DM were related to the times of administration of the medications. Other findings showed that there was good acceptability of the proposal for pharmacotherapeutic followup and that its applicability in private community pharmacies brings several benefits such as greater adherence to treatment, occurring rationally with improved clinical results and establishing a link between pharmacist and user, reaffirming the capacity pharmacist to carry out clinical activities in the private sector. This study made it clear that the practice of pharmaceutical care and good dispensing practices aimed at patients treated in drugstores can be achieved without the need for major structural changes in the establishment and without hiring additional staff.

Keywords: Type 2 diabetes mellitus; Pharmacotherapeutic follow-up; Community pharmacist.

\section{Resumen}

Esta revisión tiene como objetivo presentar evidencia sobre la relevancia de la monitorización farmacoterapéutica proporcionada a los pacientes con diabetes mellitus tipo 2 tratados en farmacias comunitarias, además de conocer el papel de los farmacéuticos comunitarios en el control de la diabetes tipo 2 e investigar los aspectos positivos de la monitorización farmacoterapéutica. dirigido a pacientes diabéticos. Se trata de una revisión de la literatura realizada mediante la búsqueda de artículos originales en bases de datos como Scielo, PubMed y Lilacs. Los critérios de inclusión fueron: publicaciones que cumplieron con la propuesta del tema, artículos completos, en português y publicados en el período de 2010 a 2019. Los criterios de exclusión fueron: estudios incompletos, que no cumplieron con el objetivo y tema de la investigación, además de libros, disertaciones y tesis. Los artículos que componen la revisión mostraron que las principales dudas de los pacientes con DM estaban relacionadas con los tiempos de administración de los medicamentos. Otros hallazgos mostraron que hubo buena aceptabilidad del seguimiento farmacoterapéutico propuesto y que su aplicabilidad en farmacias comunitarias privadas trae varios beneficios como una mayor adherencia al tratamiento, ocurriendo racionalmente con mejores resultados clínicos y estableciendo un vínculo entre farmacéutico y usuario, reafirmando la farmacéutico con capacidad para realizar actividades clínicas en el sector privado. Este estudio dejó claro que la práctica de la atención farmacéutica y las buenas prácticas de dispensación dirigida a pacientes atendidos en farmacias se puede lograr sin necesidad de grandes cambios estructurales en el establecimiento y sin contratar personal adicional.

Palabras clave: Diabetes mellitus tipo 2; Seguimiento farmacoterapéutico; Farmacéutico comunitario.

\section{Introdução}

O diabetes mellitus (DM) consiste em um distúrbio metabólico caracterizado por hiperglicemia persistente, decorrente de deficiência na produção de insulina ou na sua ação, ou em ambos os mecanismos. A hiperglicemia persistente está associada a complicações crônicas micro e macrovasculares, aumento de morbidade, redução da qualidade de vida e elevação da taxa de mortalidade. É considerado um importante problema de saúde pública em todo o mundo (Feldman, 2011; World Health Organization, 2016; International Diabetes Federation, 2017).

A classificação do DM é baseada na causa e não em seu tipo de tratamento, desta forma, os termos "insulinodependente" e "insulino-independente" não são mais utilizados para fins classificatórios. A classificação foi proposta pela World Health Organization e pela Associação Americana de Diabetes consiste de quatro classes: diabetes mellitus tipo 1 (DM1), diabetes mellitus tipo 2 (DM2), outros tipos específicos de DM e o DM gestacional. Há ainda duas categorias, classificadas como pré-diabetes, que referem-se à glicemia de jejum alterada e a tolerância à glicose diminuída, não sendo classificadas como DM, mas como fatores de risco para doenças cardiovasculares e o desenvolvimento de DM (Sociedade Brasileira de Diabetes, 2017).

O DM2 é o tipo mais relevante devido à sua importância clínico-epidemiológica, haja vista que acomete em torno de 95\% dos pacientes diagnosticados com DM (Associação Americana de Diabetes, 2013; Sociedade Brasileira de Diabetes, 2017). Este diagnóstico é respaldado ao se comprovar a carência de insulina associada a um estado de resistência do hormônio em pacientes frequentemente obesos, podendo se manifestar em qualquer faixa etária, sendo mais frequente a partir da quarta década de vida. O histórico familiar, estilo de vida pouco saudável (falta de exercícios físicos e ingestão de alimentos ricos em 
carboidratos), obesidade, envelhecimento, diabetes mellitus gestacional e a presença de componentes da síndrome metabólica: tais como hipertensão arterial e dislipidemia, são fatores de risco para o desenvolvimento de DM2 (Marinho, et al., 2013; Obreli-Neto, et al., 2013; Associação Americana de Diabetes, 2015; Sociedade Brasileira de Diabetes, 2017).

Nas últimas décadas, o número de pessoas com diagnóstico de DM cresceu e continua crescendo rapidamente ao redor do mundo. Atualmente, estima-se que a população de pacientes com diabetes seja da ordem de 425 milhões e que alcance 629 milhões em 2045, aumentando a prevalência de 8,8\% para 9,9\% no mundo. Sendo que nos EUA 25,8 milhões de pessoas são acometidas pela doença e no Brasil o DM atinge 9\% dos brasileiros, com cerca de 12,5 milhões de pessoas com o diagnóstico da doença (Whalen, et al., 2016; World Health Organization, 2016; Brasil, 2016; International Diabetes Federation, 2017).

Por conta deste cenário preocupante, em 2010 o Ministério da Saúde estabeleceu diretrizes para organização da Rede de Atenção à Saúde no Âmbito do Sistema Único de Saúde, e em 2013, instituiu a Rede de Atenção à Saúde de Pessoas com doenças crônicas, incluindo o DM. Posteriormente, estabeleceu diretrizes para a organização das linhas de cuidado da rede de atenção à saúde das pessoas com doenças crônicas nas quais atuam equipes de saúde multidisciplinares (Mendes, 2011).

Faz-se necessário repensar modelos de assistência em saúde pública com inserção das farmácias comunitárias, pois são estabelecimentos nos quais há busca por atendimento e orientações de saúde com acesso rápido e facilitado para a população, por serem muitas vezes próximas à áreas residenciais. As farmácias comunitárias facilitam o acesso aos serviços farmacêuticos para a população, realizando diariamente e legalmente os serviços, pois conta com o profissional habilitado para realizar esses serviços (Silva, 2008; Fernandes, 2014).

Neste contexto, o cuidado farmacêutico ao portador de DM2 surge então como um ramo da Assistência Farmacêutica, onde o profissional busca a melhora da qualidade de vida dos pacientes por meio de uma prática centrada no indivíduo e cuidados que devem ser tomados em relação à sua farmacoterapia. Portanto, busca-se a diminuição das taxas de mortalidade e morbidade advindas de um não controle das taxas glicêmicas (Bazotte, 2010).

Assim, pretende-se neste trabalho, por meio de revisão de literatura, descrever a atuação do farmacêutico para melhoria do prognóstico de DM2 em pacientes diabéticos atendidos em farmácias comunitárias. Para realizar a pesquisa bibliográfica foram utilizadas as principais bases de dados relacionadas ao assunto e alguns sites de associações referentes ao diabetes e ao Ministério da saúde. O objetivo desta revisão é apresentar evidências quanto à relevância do acompanhamento farmacoterapêutico prestado aos pacientes com DM2 atendidos em Farmácias comunitárias.

\section{Metodologia}

Trata-se de uma revisão de literatura, realizada por meio de busca de artigos originais em bases de dados como Scielo (Scientific Eletronic Library Online), PubMed e Lilacs (Literatura Latino-americana e do Caribe em Ciências da Saúde). De acordo Alves (2011), a pesquisa bibliográfica é "aquela desenvolvida exclusivamente a partir de fontes já elaboradas - livros, artigos científicos e publicações periódicas", tendo como vantagem cobrir uma ampla gama de fenômenos que o pesquisador não poderia contemplar diretamente.

Segundo Lakatos e Marconi (2010), a pesquisa bibliográfica abrange toda a bibliografia já publicada em relação ao tema em estudo. Sua finalidade é colocar o pesquisador em contato direto com tudo que foi escrito, dito ou filmado sobre determinado assunto. É considerada como principal tipo de fonte a imprensa escrita, em forma de jornais e revistas; as publicações em livros, publicações avulsas, assim como os meios eletrônicos.

Para o levantamento bibliográfico foram utilizados os seguintes descritores: Diabetes mellitus tipo 2, Acompanhamento farmacoterapêutico, Farmacêutico comunitário. Foram incluídas na pesquisa publicações que atenderam a proposta da temática, como artigos completos e disponíveis em língua portuguesa, publicados no período de 2010 a 2019. Os 
critérios de exclusão foram: artigos pagos, duplicados, incompletos, que não atenderam ao objetivo e temática da pesquisa, além de livros, dissertações e teses.

\section{Resultados e Discussão}

Foram selecionados três artigos que se adequaram aos critérios de inclusão estabelecidos. O desenho de estudo em todos os artigos selecionados foi o prospectivo, sendo distribuídos em países de língua portuguesa, como observado no Quadro 1 abaixo.

Quadro 1 - Artigos resultantes do levantamento bibliográfico.

\begin{tabular}{|l|l|l|l|}
\hline FONTE & TíTULO DO ARTIGO & PAÍS & DESENHO DO ESTUDO \\
\hline $\begin{array}{l}\text { Mendes, et al., } \\
2016\end{array}$ & $\begin{array}{l}\text { Autovigilância da doença e qualidade de vida dos doentes } \\
\text { diabéticos: estudo observacional em farmácias } \\
\text { comunitárias. }\end{array}$ & Portugal & Prospectivo \\
\hline $\begin{array}{l}\text { Oliveira, et al., } \\
2012\end{array}$ & $\begin{array}{l}\text { Implantação de protocolo de orientação farmacêutica } \\
\text { para indivíduos com Diabetes mellitus em farmácia } \\
\text { comunitária. }\end{array}$ & Brasil & Prospectivo \\
\hline Nunes, et al., 2012 & $\begin{array}{l}\text { Acompanhamento farmacoterapêutico de pacientes } \\
\text { diabéticos tipo 2 e fatores de risco associados. }\end{array}$ & Brasil & Prospectivo \\
\hline
\end{tabular}

Fonte: Autores.

No estudo de Oliveira et al. (2012), indivíduos maiores de 18 anos portando prescrições de medicamentos antidiabéticos orais foram convidados a participar do protocolo de orientação mínima. Procedeu-se à análise da prescrição e orientações relativas ao uso de medicamentos. Avaliou-se a aceitabilidade da proposta e o tempo para análise da prescrição, dispensação e aconselhamento. Foram realizados 40 atendimentos (74\% das abordagens), com tempo médio de 5 minutos, enquanto 22,5\% precisaram de 1,8 minutos adicionais. As principais dúvidas foram relacionadas aos horários de administração dos medicamentos. De acordo com os achados destes autores, houve boa aceitabilidade da proposta, sendo a pressa o principal motivo para as recusas. Ademais, o protocolo mostrou-se adequado para o esclarecimento de dúvidas sobre o uso de medicamentos e a doença, não exigindo alterações estruturais ou de recursos humanos no estabelecimento. Sua implantação foi facilitada por ser focado em uma única condição clínica.

Nunes et al. (2012) desenvolveram um estudo em farmácias comunitárias no qual 58 voluntários responderam a questionários semiestruturados durante sete entrevistas agendadas. A análise dos dados identificou que a hipertensão está presente na maioria dos pacientes diabéticos e o tabagismo e o etilismo não se mostraram evidentes na população estudada. A forma de intervenção mais praticada foi a do tipo farmacêutico-paciente. A prática do AF firmou-se influente na melhoria da qualidade de vida dos pacientes diabéticos, através da identificação e esclarecimento de dúvidas relacionadas aos medicamentos, bem como de orientações sobre os aspectos relacionados à melhoria da qualidade de vida como o uso racional dos fármacos.

Na pesquisa de Mendes et al. (2016) 211 farmácias aceitaram participar no estudo. Foram recrutados 1.479 pacientes diabéticos adultos, sendo $53,7 \%$ do sexo feminino e com idade média de 64,1 anos. Neuropatia (27,3\%), retinopatia $(22,4 \%)$ e nefropatia $(11,9 \%)$ foram as complicações associadas à diabetes mais frequentemente reportadas. Na maioria dos doentes, a autovigilância da glicemia foi referida com frequência superior a uma ou 2 vezes por mês. Em cerca de $30 \%$ dos casos, a autovigilância dos pés nunca ou raramente foi efetuada e $27 \%$ dos doentes declararam nunca ter feito uma consulta de oftalmologia. Análises à glicemia e hemoglobina glicosilada foram realizadas anualmente em $25 \%$ dos participantes. A maioria dos pacientes fazia uso antidiabéticos orais.

Esses achados corroboram com as recomendações propostas por Pereira et al. (2017), em que se pontua que o 
emprego do seguimento farmacoterapêutico (SFT) em farmácias comunitárias privadas traz diversos benefícios como maior adesão ao tratamento, ocorrendo de forma racional com melhoria dos resultados clínicos e estabelecimento de vínculo entre farmacêutico e usuário, reafirmando a capacidade do farmacêutico para a realização das atividades clínicas no setor privado.

Resultados positivos obtidos com o SFT também foram demonstrados no estudo de Zubioli et al. (2013), que avaliou um programa de atenção farmacêutica que aumentou a eficácia da farmacoterapia no tratamento de pacientes portadores de DM2, além da redução de riscos de complicações de DM e outras doenças crônicas. Foram acompanhados diabéticos que possuíam glicemia de jejum acima de $140 \mathrm{mg} / \mathrm{dL}$ ou hemoglobina glicosilada considerada alta (acima de 7\%). O programa incluía estratégias de vários SFT, incluindo o método Dáder, onde avaliava o estio de vida, a farmacoterapia e a monitorização de teste laboratoriais e sinais vitais. Essa rotina foi feita quatro vezes durante um ano. A cada consulta foi proposta uma educação contínua ao paciente em relação aos cuidados com a saúde e o uso dos seus medicamentos.

Braz et al. (2017), relatam a importância do profissional farmacêutico no acompanhamento aos pacientes em todas as etapas da farmacoterapia para melhorar a adesão farmacoterapêutica, com atenção especial às orientações dadas aos pacientes no momento da dispensação, pois pacientes insatisfeitos com as orientações recebidas podem ficar com dúvidas e consequentemente não aderirem ao tratamento de forma adequada. No estudo dos autores supracitados, $78 \%$ dos pacientes possuíam dúvidas quanto ao medicamento prescrito pelo médico, verificando que os pacientes têm a necessidade de saber mais sobre o seu tratamento e medicamentos.

Dessa forma, o farmacêutico deverá prestar serviços que, promovam uma farmacoterapia segura aos pacientes da farmácia comunitária, como: oferecer informações sobre cuidados com a saúde; orientar sobre a natureza, causas e consequências do DM e a importância do tratamento; dispensar o medicamento de forma ativa mediante prescrição; verificar os níveis de glicemia; identificar os eventos adversos, orientando-os como minimizar e prevenir tais reações; avaliar a adesão ao tratamento medicamentoso e não medicamentoso e intervir sobre os problemas de saúde conforme sua autonomia profissional (Brasil, 2011).

Entretanto, existem desafios a serem superados para implantação do acompanhamento farmacoterapêutico em farmácias comerciais, dentre os quais destaca-se o fato de que parte da população enxerga a farmácia comunitária privada como um ambiente apenas comercial e não de saúde. Isso ocasiona dificuldades para construção de vínculos entre o farmacêutico e paciente, o que limita a possibilidade de intervenções farmacêuticas (Pereira, et al., 2017).

\section{Conclusão}

Este estudo tornou evidente que a prática da assistência farmacêutica e das boas práticas de dispensação direcionadas aos pacientes atendidos em drogarias pode ser alcançada sem a necessidade de grandes alterações estruturais no estabelecimento e sem a contratação adicional de funcionários. Colocar em prática protocolos de acompanhamento farmacoterapêutico, relatados nos artigos presentes nesta revisão, mostrou-se viável e adequado para promover o uso correto de medicamentos, horários de administração e também na identificar falhas no acompanhamento da doença, principalmente a ausência do controle.

É notório o impacto positivo do cuidado farmacêutico, sendo assim este profissional mostra-se um relevante agente para promoção do uso racional de medicamentos em todos os cenários em que estiver inserido, principalmente nas drogarias. As organizações de saúde brasileiras têm assumido esse papel de destaque no processo de construção de um modelo brasileiro de cuidados farmacêuticos e, diante desta perspectiva é imprescindível que os farmacêuticos comunitários promovam a implementação dos cuidados farmacêuticos em seus ambientes de trabalho. 
Research, Society and Development, v. 10, n. 8, e9010817150, 2021

(CC BY 4.0) | ISSN 2525-3409 | DOI: http://dx.doi.org/10.33448/rsd-v10i8.17150

\section{Referências}

American Diabetes Association. (2013). Standards of Medical Care in Diabetes. Diabetes Care, 36, S11-S66. 10.2337/dc13-S011.

American Diabetes Association. (2015). Standards of medical Care in Diabetes. Diabetes Care, 38, S4. 10.2337/dc15-S003

Bazotte, R. B. (2012). Paciente diabético: Cuidados Farmacêuticos. MedBook.

Brasil. (2011). Conselho Regional de Farmácia do Estado de São Paulo. Fascículo VII - Manejo do tratamento de Pacientes com diabetes, 78 p.

Brasil. (2016). Conselho Federal de Farmácia. Serviços Farmacêuticos Diretamente Destinados ao Paciente, à Família e à Comunidade: Contextualização e Arcabouço Conceitual. Programa de Suporte ao Cuidado Farmacêutico na Atenção à Saúde. 200 p.

Braz, A. L., Ferreira, E. C., Guedes, D. N., Costa, K. V. M. C., Coreia, N. A., Albuquerque, K. L. G. (2017). Atenção farmacêutica em pacientes hipertensos do Hospital Universitário Lauro Wanderley. Revista de Ciências Médicas e Biológicas, 16(1), 45-51.

Feldman, F. G. S. (2011). Avaliação da atenção farmacêutica para pacientes diabéticos. Trabalho de Conclusão de Curso, Fundação Oswaldo Cruz.

Ferreira, V. L. (2014). A importância do seguimento farmacoterapêutico na saúde: uma revisão da literatura. Trabalho de conclusão de curso, Universidade Federal da Paraíba.

International Diabetes Federation. (2017). IDF Diabetes Atlas Eighth Edition. Bruxelas, Bélgica: International Diabetes Federation.

Lakatos, E.M. \& Marconi, M.A. (2010). Fundamentos da metodologia científica. Atlas.

Mendes, E. V. (2011). As redes de atenção à saúde. Organização Pan-Americana da Saúde.

Mendes, I., Guedes, S., Guerreiro, J. P., Inês, M., Sousa, A., \& Miranda, A. (2016). Autovigilância da doença e qualidade de vida dos doentes diabéticos: estudo observacional em farmácias comunitárias, Revista Portuguesa de Saúde Pública, 34(1):11-19.

Nunes, L. M. N. et al. (2012). Acompanhamento farmacoterapêutico de pacientes diabéticos tipo 2 e fatores de risco associados. Revista Brasileira de Farmácia, 93(2): 196-203.

Oliveira, T. C. A. et al. (2012). Implantação de protocolo de orientação farmacêutica para indivíduos com Diabetes mellitus em farmácia comunitária. Revista Brasileira de Farmácia, 93(3): 379-384.

Pereira, M. G., Prado, N. M. B. L., \& Krepsky, P. B. (2017). Resultados de Seguimento Farmacoterapêutico a pacientes hipertensos em farmácia comunitária privada na Bahia. Revista Baiana de Saúde Pública, 41(2).

Silva, E. V., Naves, J. O. S. \& Vidal, J. (2008). O papel do farmacêutico comunitário no aconselhamento ao paciente. Farmacoterapêutica, 8(4-5), 2-6.

Obreli-Neto, P. R., Baldoni, A. O. \& Guidoni, C. M. (2013). Farmacoterapia: Guia terapêutico de doenças mais prevalentes. Pharmabooks.

World Health Organization. (2016). Global report on diabetes. Geneva, Switzerland: WHO Library Cataloguing-in-Publication Data.

Whalen, K., Finkel, R. \& Panavelil, T. A. (2016). Farmacologia ilustrada. Artmed.

Zubioli, A., Silva, M. A. R. C. P. Da, Tasc, R. S., Curi, R. \& Bazotte, R. B. (2013). Pharmaceutical consultation as a tool to improve health outcomes for patients with type 2 diabetes. Brazilian Journal of Pharmaceutical Sciences, 49(1). 